\title{
Two-Year Trends of Taxane-Induced Neuropathy in Women Enrolled in a Randomized Trial of Acetyl-L- Carnitine (SWOG S0715)
}

\author{
Dawn L. Hershman, Joseph M. Unger, Katherine D. Crew, Cathee Till, \\ Heather Greenlee, Lori M. Minasian, Carol M. Moinpour, Danika L. Lew, \\ Louis Fehrenbacher, James L. Wade III, Siu-Fun Wong, Michael J. Fisch, \\ N. Lynn Henry, Kathy S. Albain
}

Affiliations of authors: Columbia University Medical Center, New York, NY (DLH, KDC, HG); SWOG Statistical Center, Seattle, WA (JMU, CT, DLL); Fred Hutchinson Cancer Research Center, Seattle, WA (JMU, CT, CMM, DLL); Community Oncology and Prevention Trials Research Group, Division of Cancer Prevention, National Cancer Institute, Bethesda, MD (LMM); Kaiser Permanente Northern California, Vallejo, CA (LF); Central Illinois CCOP/Cancer Care Specialists of Central Illinois, Decatur, IL (JLWIII); Loma Linda University School of Pharmacy, Loma Linda, CA (SFW); AIM Specialty Health, Chicago, IL (MJF); Huntsman Cancer Institute, Salt Lake City, UT (NLH); Loyola University Chicago Stritch School of Medicine, Cardinal Bernardin Cancer Center, Maywood, IL (KSA)

Correspondence to: Dawn L. Hershman, MD, MS, Columbia University, 161 Fort Washington Avenue, 10-1068, New York, NY 10032 (e-mail: dlh23@columbia.edu).

\begin{abstract}
Background: Chemotherapy-induced peripheral neuropathy (CIPN) is a common and disabling side effect of taxanes. AcetylL-carnitine (ALC) was unexpectedly found to increase CIPN in a randomized trial. We investigated the long-term patterns of CIPN among patients in this trial.

Methods: S0715 was a randomized, double-blind, multicenter trial comparing ALC (1000 mg three times a day) with placebo for 24 weeks in women undergoing adjuvant taxane-based chemotherapy for breast cancer. CIPN was measured by the 11-item neurotoxicity (NTX) component of the FACT-Taxane scale at weeks 12, 24, 36, 52, and 104. We examined NTX scores over two years using linear mixed models for longitudinal data. Individual time points were examined using linear regression. Regression analyses included stratification factors and the baseline score as covariates. All statistical tests were twosided.

Results: Four-hundred nine subjects were eligible for evaluation. Patients receiving ALC had a statistically significantly $(\mathrm{P}=.01)$ greater reduction in NTX scores (worse CIPN) of -1.39 points (95\% confidence interval [CI] $=-2.48$ to -0.30$)$ than the placebo group. These differences were particularly evident at weeks $24(-1.68,95 \% \mathrm{CI}=-3.02$ to -0.33$), 36(-1.37,95 \% \mathrm{CI}=-2.69$ to -0.04$)$, and $52(-1.83,95 \% \mathrm{CI}=-3.35$ to -0.32$)$. At 104 weeks, $39.5 \%$ on the ALC arm and $34.4 \%$ on the placebo arm reported a five-point (10\%) decrease from baseline. For both treatment groups, 104-week NTX scores were statistically significantly different compared with baseline $(P<.001)$.

Conclusions: For both groups, NTX scores were reduced from baseline and remained persistently low. Twenty-four weeks of ALC therapy resulted in statistically significantly worse CIPN over two years. Understanding the mechanism of this persistent effect may inform prevention and treatment strategies. Until then, the potential efficacy and harms of commonly used supplements should be rigorously studied.
\end{abstract}


Chemotherapy-induced peripheral neuropathy (CIPN) is a common and disabling side effect of some widely used anticancer agents. To date, despite 48 randomized trials, there are no agents recommended for the prevention of CIPN (1). There is a moderate recommendation for the treatment of painful CIPN with duloxetine. We previously reported the unexpected outcomes of a randomized double-blind placebo-controlled trial of acetyl-L-carnitine (ALC) for the prevention of taxane-induced neuropathy in women undergoing adjuvant breast cancer therapy (S0715) (2). Despite the supportive evidence from preclinical and phase II studies that ALC may be effective for both the treatment and prevention of taxane-induced symptoms of CIPN, in this large placebo-controlled trial, we found that ALC actually increased CIPN and decreased functional status relative to the placebo at 24 weeks (2).

The symptoms of CIPN may have a waxing and waning course (3). Up to $80 \%$ of patients treated with taxanes report some neuropathy, and about $25 \%$ to $30 \%$ report severe neuropathy up to two years following treatment (3-5). Several crosssectional studies of patients treated with taxanes reported that persistent CIPN symptoms were associated with increased risk of falls, greater disability, and increased psychosocial distress $(6,7)$. However, cross-sectional studies can be confounded by reporting bias, and most prospective studies characterizing the syndrome have been small with heterogeneous treatment regimens, nonuniform outcome measures, and/or short-term follow-up $(3,8)$. The goal of this analysis was to examine long-term CIPN outcomes, out to two years, and to better characterize the clinical phenotype of CIPN.

\section{Methods}

\section{Study Oversight}

The study, registered with ClinicalTrials.gov (NCT00775645), was activated in September 2009 and closed to accrual February 2011. Patients were informed of the investigational nature of the study and signed informed consent. The study was conducted after appropriate approval by individual institutional review boards in compliance with the provisions of the Declaration of Helsinki and Good Clinical Practice guidelines.

\section{Patient Characteristics}

Women older than age 18 years with a history of stage I-III breast cancer scheduled to undergo adjuvant taxane-based chemotherapy with one of the following standard regimens were included: weekly paclitaxel $80 \mathrm{mg} / \mathrm{m}^{2}$ for 12 cycles; biweekly paclitaxel $175 \mathrm{mg} / \mathrm{m}^{2}$ for four cycles; biweekly paclitaxel 175 $\mathrm{mg} / \mathrm{m}^{2}$ for six cycles; every-three-week docetaxel $75 \mathrm{mg} / \mathrm{m}^{2}$ for four cycles; or every-three-week docetaxel $75 \mathrm{mg} / \mathrm{m}^{2}$ for six cycles. Patients were required to have a Zubrod performance status of $0-2$. Serum creatinine of 2.5 or more times the institutional upper limit of normal was required. Patients with a history of diabetes, prior history of peripheral neuropathy from other causes, or with a seizure disorder were excluded. Patients on any of the following medications used to treat CIPN were excluded: vitamin E, glutamine, gabapentin, nortriptyline, amitriptyline, or duloxetine $\mathrm{HCl}$, and other nutritional supplements used to treat CIPN.

\section{Study Design}

A randomized, double-blind, multicenter trial comparing $3000 \mathrm{mg} / \mathrm{d}$ (six capsules) of ALC (Thorne Research, Inc., Dover, ID) with matching placebo daily for 24 weeks was conducted. Each active capsule contained $590 \mathrm{mg}$ of acetyl-Lcarnitine HCL and $10 \mathrm{mg}$ of cellulose. The $590 \mathrm{mg}$ of acetyl-Lcarnitine HCL provides $500 \mathrm{mg}$ of ALC. Each placebo capsule contained $600 \mathrm{mg}$ of cellulose. Patient random assignment was stratified by planned taxane-based chemotherapy treatment and age ( $<60$ vs $\geq 60$ years). The study drug was initiated at the start of taxane chemotherapy. Following completion of the study, the capsules were retested for stability and composition.

\section{Outcome Measures}

Patients were assessed at baseline (prior to starting taxane and ALC/placebo) for peripheral neuropathy using the 11item Neurotoxicity component of the Functional Assessment of Cancer Therapy-Taxane (FACT-NTX) symptom module as a continuous measure (range $=0-44$ ). A lowering in the FACT-NTX score (worse CIPN) of more than $10 \%$ or five points is considered clinically significant (9). We also conducted a sensitivity analysis evaluating a 10-point decrease in FACTNTX score from baseline. For secondary objectives, functional status was measured with the FACT-Taxane Trial Outcome Index (TOI) score (range $=0-120$ ) (9). Fatigue was measured by the 13-item Functional Asessment of Chronic Illness Therapy (FACIT)-Fatigue scale (range $=0-52$ ). For each instrument, lower scores reflected worse symptoms $(9,10)$. Patients were assessed at baseline and weeks 12, 24, 36,52 , and 104 . We previously reported the results at weeks 12 and 24 (2).

\section{Statistical Analysis}

Baseline demographic factors were compared between treatment groups using chi-square tests for categorical variables and $t$ tests for continuous variables (all two-sided tests). We examined the effect of ALC on CIPN, as measured by FACT-NTX score, over two years using linear mixed models for longitudinal data to account for correlated outcomes within patients over time. The outcome is NTX score at each time point except baseline. We specified a random intercept and slope for time and adjusted for the protocol specified stratification factors (planned taxane-based chemotherapy and age [ $<60$ vs $\geq 60$ years]) as covariates. For each model, we also adjusted for the baseline NTX score to account for any residual imbalance between intervention groups. Exploratory plots of the outcomes over time suggested a nonlinear relationship; thus, for each outcome, we modeled outcomes over time using a linear time variable and a quadratic time variable. The residual errors were determined to be normally distributed. Other patient-reported secondary outcomes (FACT-Trial Outcome Index [TOI], FACIT-Fatigue) were examined in a similar fashion. Simple linear models were used to describe potential differences in outcomes at individual assessment times.

To better characterize the clinical phenotype of CIPN, we examined the relationship between one- and two-year FACT-NTX scores and baseline factors, including age, race, ethnicity, weight, prior mastectomy, planned treatment regimen, performance status, and receipt of G-CSF (granulocyte 


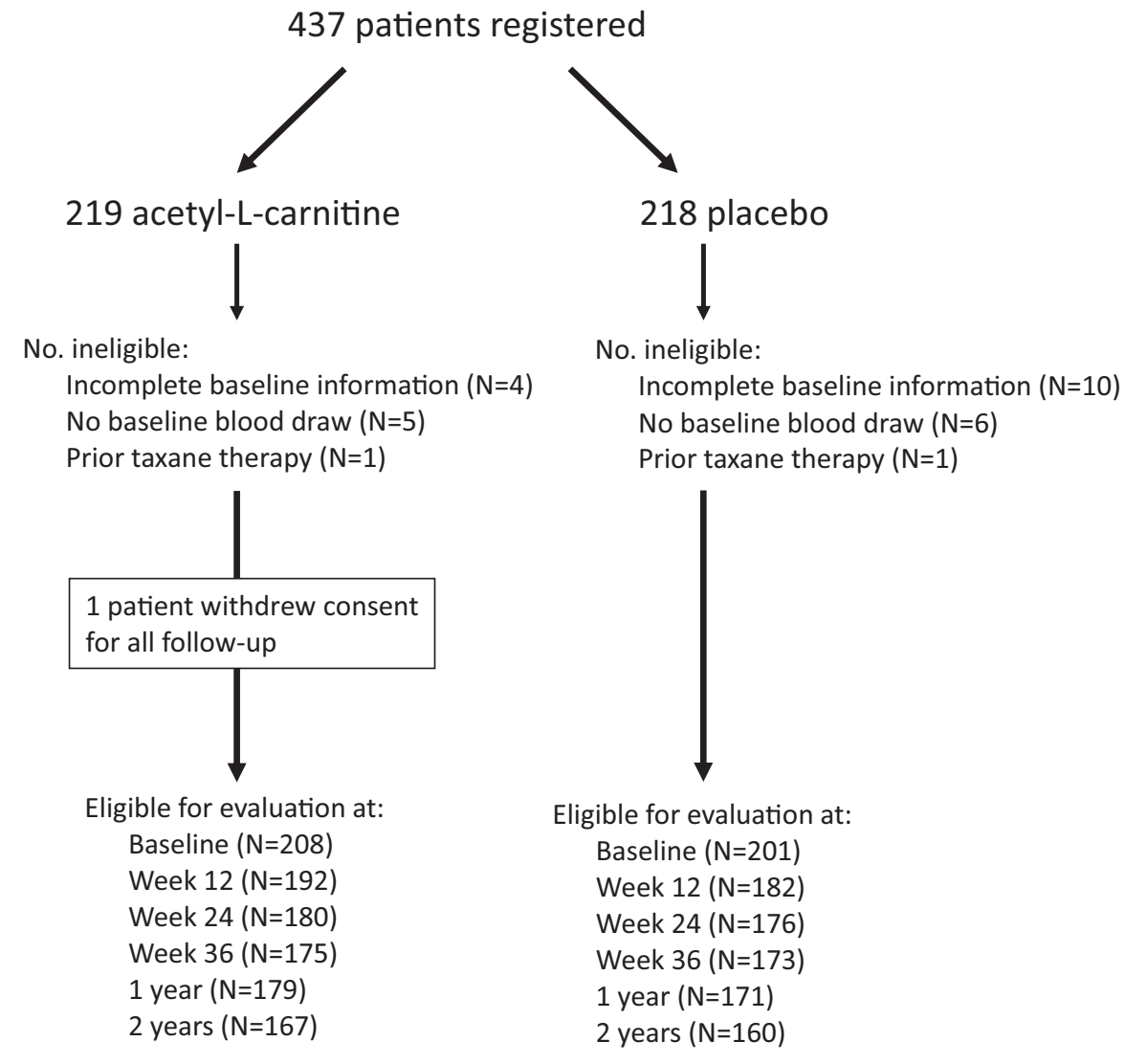

Figure 1. Consort diagram.

colony-stimulating factor). Predictive factors were identified by testing the interaction of each baseline factor with treatment arm. If no interaction was evident $(P>.05)$, then data were combined across arms, and the potential prognostic effects of the different factors were examined, adjusting for treatment arm as a covariate. Logistic regression was used to explore associations between these predictive factors and risk of a five-point decrease in FACT-NTX at year 1 and year 2 .

The study was designed to detect a minimally important difference of three points in the FACT-NTX score (10). We prespecified a test of the intervention effect on the FACT-NTX over the full two years of assessments as the single primary examination, at a two-sided alpha of .05. All other tests were considered exploratory, with two-sided $P$ values provided. All models were fit in R version 3.3.1 (R Core Team, 2013) (11) and SAS version 9.4 (SAS Institute, Inc., Cary, NC).

\section{Results}

\section{Patient Characteristics}

SWOG S0715 registered 437 patients, of whom 409 had both baseline and first follow-up outcome measures, including 208 assigned to receive ALC and 201 to placebo (Figure 1). Patient characteristics were well balanced by intervention assignment for all factors, as shown in Table 1. Patients were predominantly younger than 60 years; $9.3 \%$ percent of all included patients were black, and $4.9 \%$ were Asian. Twenty point five percent of patients had stage III disease.

\section{Long-term Outcomes of Acetyl-L-carnitine Use}

The number of patients with available FACT-NTX scores diminished over time, but was similar between arms. Missing outcome data at each time point did not exceed $5 \%$ in either treatment group and did not differ over time by group. The number of evaluable patients in the study also did not differ statistically significantly over time by arm. Mean FACT-NTX scores in both treatment groups decreased over time and were statistically significantly different at two years compared with baseline $(P<.001)$ (Figure 2).

We found no evidence that the trajectories over time for the FACT-NTX score differed by arm using either a quadratic $(P=.78)$ or linear interaction $(P=.91$; data not shown). However, there was evidence that the trajectories for both arms varied over time in a quadratic fashion $(P=.002)$. Thus, the final model included both linear and quadratic variables for time, but no interaction between time and intervention arm. In this model setting, the results for the primary outcome of interest, NTX, show a statistically significant $(P=.01)$ average difference of -1.39 (95\% confidence interval $[\mathrm{CI}]=-2.48$ to -0.30 ) between treatment groups, with the ALC group having lower scores (worse CIPN) on average than the placebo group (Table 2). These differences were particularly evident at week $24(-1.68,95 \%$ $\mathrm{CI}=-3.02$ to -0.33$)$, week $36(-1.37,95 \% \mathrm{CI}=-2.69$ to -0.04$)$, and week $52(-1.83,95 \% \mathrm{CI}=-3.35$ to -0.32$)$. The percentage of patients with a five-point (10\%) decrease from baseline at each time point is shown in Figure 3. At 104 weeks, 39.5\% on 
Table 1. Baseline demographic factors

\begin{tabular}{|c|c|c|c|}
\hline Demographic factors & $\begin{array}{c}\text { ALC } \\
(n=208)\end{array}$ & $\begin{array}{l}\text { Placebo } \\
(\mathrm{n}=201)\end{array}$ & $P^{*}$ \\
\hline Age, mean (SD), y & $53.3(9.9)$ & $51.9(10.9)$ & .16 \\
\hline Age < 60 y, No. (\%) & $150(72.1)$ & $146(72.6)$ & .91 \\
\hline Race, No. (\%) & & & .32 \\
\hline White & $160(76.9)$ & $165(82.1)$ & \\
\hline Black & $18(8.7)$ & $20(10.0)$ & \\
\hline Asian & $12(5.8)$ & $8(4.0)$ & \\
\hline Pacific Islander & $4(1.9)$ & $3(1.5)$ & \\
\hline Native American & $2(1)$ & $0(0.0)$ & \\
\hline Unknown & $12(5.8)$ & $5(2.5)$ & \\
\hline Hispanic, No. (\%) & & & .47 \\
\hline Yes & $21(10.1)$ & $15(7.5)$ & \\
\hline No & $181(87.0)$ & $177(88.0)$ & \\
\hline Unknown & $6(2.9)$ & $9(4.5)$ & \\
\hline Taxane regimen, No. (\%) & & & .91 \\
\hline Weekly paclitaxel, $12 \mathrm{wk}$ & $73(35.1)$ & $68(33.8)$ & \\
\hline $\begin{array}{l}\text { Biweekly paclitaxel } \\
\text { for } 4 \text { cycles, } 8 \mathrm{wk}\end{array}$ & $48(23.0)$ & $51(25.4)$ & \\
\hline $\begin{array}{l}\text { Biweekly paclitaxel } \\
\text { for } 6 \text { cycles, } 12 \mathrm{wk}\end{array}$ & $3(1.4)$ & $5(2.5)$ & \\
\hline $\begin{array}{l}\text { Every-3-wk docetaxel } \\
\text { for } 4 \text { cycles, 12-wk TC }\end{array}$ & $51(24.5)$ & $48(23.9)$ & \\
\hline $\begin{array}{l}\text { Every-3-wk docetaxel for } \\
\quad 6 \text { cycles, 18-wk TAC or TC }\end{array}$ & $33(15.9)$ & $29(14.4)$ & \\
\hline Performance Status, No. (\%) & & & .86 \\
\hline 0 & $156(75)$ & $146(73)$ & \\
\hline 1 & $51(25)$ & $54(27)$ & \\
\hline 2 & $1(<1)$ & $1(<1)$ & \\
\hline Stage, No. (\%) & & & .82 \\
\hline I & $57(27.4)$ & $50(25.0)$ & \\
\hline II & $110(52.9)$ & $107(53.5)$ & \\
\hline III & $41(19.7)$ & $43(21.5)$ & \\
\hline
\end{tabular}

*Baseline factors were compared by treatment groups using chi-square tests for categorical variables and $t$ tests for continuous variables. Tests were two-sided. $\mathrm{A}=$ doxorubicin; $\mathrm{C}=$ cyclophosphamide; $\mathrm{T}=$ docetaxel.

the ALC arm and $34.4 \%$ on the placebo arm reported a five-point decrease. About $19.0 \%$ on the ALC and $18.0 \%$ on placebo arm reported persistent symptoms (10-point [20\%] decrease in NTX) at 104 weeks.

Using the same model structure for the FACT-TOI and FACIT-Fatigue end points, there was no evidence of a statistically significant difference by arm in score results over time (Table 2), although the trend in FACT-TOI results was similar to that of the FACT-NTX scores (Supplementary Figures 1 and 2, respectively).

We evaluated use of other medications and supplements that have been used to treat CIPN (Supplementary Figure 3, available online). No differences by treatment at any time point were observed (data not shown). Of note, antidepressant use and omega- 3 fatty acid (ie, fish oil) use increased slightly over time, and opioid use decreased over time.

\section{Predictors of Persistent Neuropathy}

Women age 60 years or older had an increased risk of worse neuropathy symptoms compared with women younger than age 60 years at year 1 (odds ratio $[\mathrm{OR}]=1.74,95 \% \mathrm{CI}=1.07$ to $2.82, P=.02)$ and year $2(\mathrm{OR}=1.67,95 \% \mathrm{CI}=1.02$ to 2.73 ,
$P=.04)$ (Table 3). The association of weight and long-term FACT-NTX scores differed by arm at years $1\left(P_{\text {interaction }}=.06\right)$ and $2\left(P_{\text {interaction }}=.047\right)$. On the ALC arm, weight was associated with a $14 \%$ increased risk of worsening neuropathy per $5 \mathrm{~kg}$ of weight at year 1 (OR $=1.14,95 \% \mathrm{CI}=1.05$ to $1.24, \mathrm{P}=$ .002 ) and a $16 \%$ increased risk of worsening neuropathy per 5 $\mathrm{kg}$ of weight at year $2(\mathrm{OR}=1.16,95 \% \mathrm{CI}=1.06$ to $1.26, \mathrm{P}<$ .001). There was no statistically significant association between weight and decreased FACT-NTX on the placebo arm at either time point. There was no evidence that any other baseline factor was related to the risk of worsening neuropathy symptoms at either time point for either the placebo or the ALC arm.

\section{Discussion}

In this large randomized placebo-controlled trial, we showed that ALC treatment resulted in worsening of CIPN symptoms compared with placebo that persisted over two years. In addition, we showed that two years after taxane initiation, among those treated on the placebo arm, $34.4 \%$ had a persistent fivepoint decrease from baseline, and $18.0 \%$ had a persistent $10-$ point decrease from baseline on the FACT-NTX score. Older age was the only baseline factor that was associated with persistent symptoms in the placebo arm.

We were initially surprised to find that ALC increased CIPN during the 24-week intervention, and continue to be surprised that the difference between arms persisted throughout the twoyear period following ALC discontinuation. The reasons for this are unclear. ALC is an antioxidant, which may be contributing to a protective effect against oxidative stress (12). Reactive oxygen species (ROS) can cause damage to DNA, which can result in tumor and normal cell death; however, they may also block treatment-generated ROS, leading to lower levels of cell damage $(12,13)$. We have previously reported that patients who initiated antioxidant therapy during chemotherapy were more likely to develop CIPN six months after initiating treatment (14). It is also possible that early development of symptoms is associated with prolonged duration of CIPN. The results suggest that more effort should be placed on ensuring safety and efficacy for over-thecounter supplements that are commonly used during cancer treatment.

Most clinical trials report adverse CIPN events during treatment, although few report the presence of persistent symptoms following treatment, and most have not used patient-reported outcomes. Therefore, the literature on long-term CIPN is very limited. Several cross-sectional studies conducted in cancer survivors report the prevalence of CIPN symptoms ranging from $30 \%$ to $80 \%$ depending on the population studied $(6,15,16)$, the measure used to assess CIPN, the timing of the measure following treatment, and the chemotherapy drug administered. However, without baseline assessment, it is difficult to determine if any of these symptoms were present prior to chemotherapy, or whether the findings may have been influenced by selection and recall bias. Several of these cross-sectional studies report a relationship between worse scores on CIPN patient-reported outcomes and decreased quality of life, increased risk of falls, and increased functional impairment $(6,7)$.

Only a few prospective CIPN cohort studies that enrolled subjects prior to treatment initiation have been conducted $(3,17,18)$. Many of these studies suffer from small sample size, 


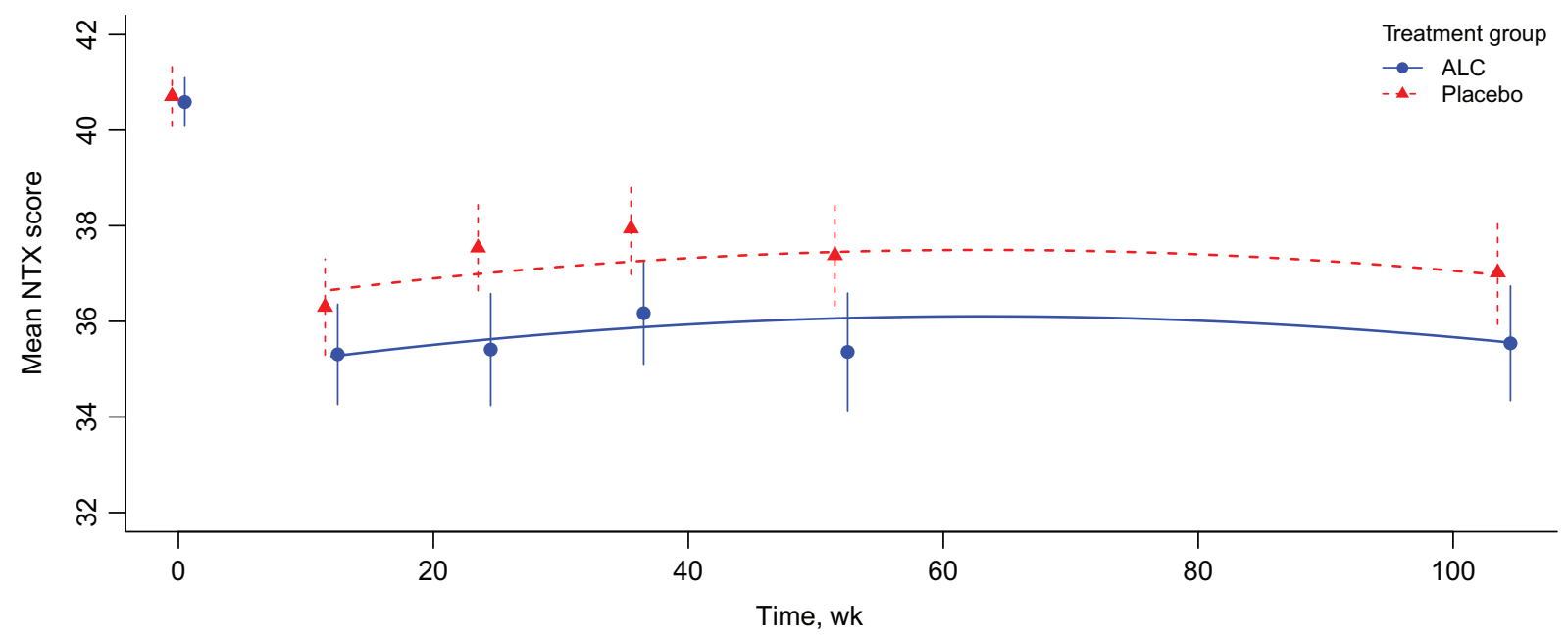

Figure 2. Observed and model-fitted mean Neurotoxicity component of the Functional Assessment of Cancer Therapy-Taxane score over time among participants randomly assigned to acetyl-L-carnitine and placebo. Points represent observed mean values at each time. Error bars represent $95 \%$ confidence intervals. Lines represent the fitted values based on linear mixed model results. ALC = acetyl-L-carnitine.

Table 2. Results of multivariable longitudinal (linear mixed) model and simple linear model regression analyses

\begin{tabular}{|c|c|c|c|c|c|c|}
\hline \multirow[b]{3}{*}{ Outcome } & \multicolumn{6}{|c|}{ Coefficient for treatment term (acetyl-L-carnitine vs placebo) } \\
\hline & \multirow{2}{*}{$\begin{array}{l}\text { Linear mixed } \\
\text { model result* } \\
12 \text { to } 104 \mathrm{wk}\end{array}$} & \multicolumn{5}{|c|}{ Linear models $\dagger$} \\
\hline & & Week 12 & Week 24 & Week 36 & Week 52 & Week 104 \\
\hline \multicolumn{7}{|l|}{ FACT-NTX } \\
\hline $\begin{array}{l}\text { Coefficient } \\
\text { (95\% CI) }\end{array}$ & $-1.39(-2.48$ to -0.30$)$ & $-0.91(-2.21$ to 0.39$)$ & $-1.68(-3.02$ to -0.33$)$ & $-1.37(-2.69$ to -0.04$)$ & $-1.83(-3.35$ to -0.32$)$ & $-1.03(-2.51$ to 0.44$)$ \\
\hline$P$ & .01 & .17 & .02 & .04 & .02 & .17 \\
\hline \multicolumn{7}{|l|}{ FACT-TOI§ } \\
\hline $\begin{array}{l}\text { Coefficient } \\
\quad(95 \% \text { CI })\end{array}$ & $-1.89(-4.36$ to 0.57$)$ & -0.02 (-2.87 to 2.83$)$ & $-3.24(-6.25$ to -0.23$)$ & $-2.18(-5.32$ to 0.95$)$ & $-3.33(-6.63$ to -0.03$)$ & -2.94 (-6.31 to 0.43$)$ \\
\hline$P$ & .13 & .99 & .04 & .17 & .05 & .09 \\
\hline \multicolumn{7}{|l|}{ FACIT-Fatigue $\|$} \\
\hline $\begin{array}{c}\text { Coefficient } \\
\quad(95 \% \text { CI) }\end{array}$ & -0.05 (-1.51 to 1.42$)$ & 1.38 (-0.57 to 3.33$)$ & $-0.43(-2.29$ to 1.43$)$ & $-0.17(-2.15$ to 1.81$)$ & $-0.48(-2.45$ to 1.48$)$ & -1.83 (-3.83 to 0.17$)$ \\
\hline$P$ & .95 & .17 & .65 & .87 & .63 & .07 \\
\hline
\end{tabular}

*For the linear mixed models, the outcome is NTX score at each time point except baseline, using a random intercept and slope for time, and adjusted for planned taxane-based chemotherapy, age ( $<60$ vs $\geq 60$ years), and baseline NTX score. P values are two-sided. CI = confidence interval; FACIT-Fatigue = Functional Asessment of Chronic Illness Therapy; FACT-NTX = Neurotoxicity component of the Functional Assessment of Cancer Therapy-Taxane; FACT-TOI = Functional Assessment of Cancer Therapy-Trial Outcome Index.

$\dagger$ For the linear models, the outcome is NTX score at each respective time point, and each model is adjusted for planned taxane-based chemotheraphy, age ( $<60$ vs $\geq 60$ years), and baseline NTX score. $P$ values are two-sided.

$\neq$ FACT-Taxane neurotoxicity (range $=0-44$ ).

$\S$ FACT-Taxane-TOI score (range $=0-120$ ).

$\|$ FACIT-Fatigue scale (range $=0-52$ ).

short follow-up, and treatment heterogeneity. Members of our group conducted a prospective study among 50 women with breast cancer treated with adjuvant taxane therapy. Patients were assessed at baseline, following therapy, and three, six, nine, and 12 months after completing therapy. The mean scores on the FACT-NTX decreased from 37.5 to 28.7 post-treatment $(P=.0002)$, and remained low 12 months after treatment. In a prior study, we found that the changes in hand/foot numbness/ discomfort were statistically significantly associated with change in vibration threshold measured with quantitative sensory testing (3). In the current study, we found similar results.
For comparison purposes, among women on the placebo arm, we found that mean FACT-NTX decreased from 41 to 37 at one year and remained at 37 at two years. Similarly, the percentage of patients with a five-point decrease from baseline was similar at years 1 and 2 .

Prior studies have examined factors associated with maximum grade or symptoms of neuropathy, but few have evaluated factors associated with persistent symptoms (19-25). Some studies suggest that race, age, and obesity may be risk factors for CIPN; however, other studies have not confirmed these findings (19-25). In the current study, age was associated with 
A 70 5 -point change

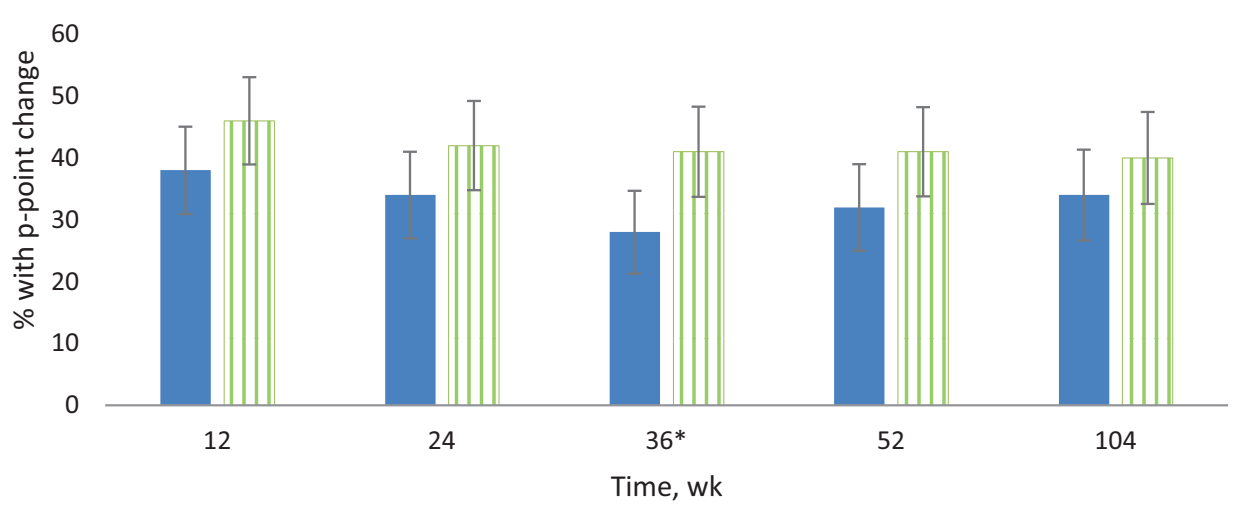

Control $\square \mathrm{ALC}$

B

70

10-point change

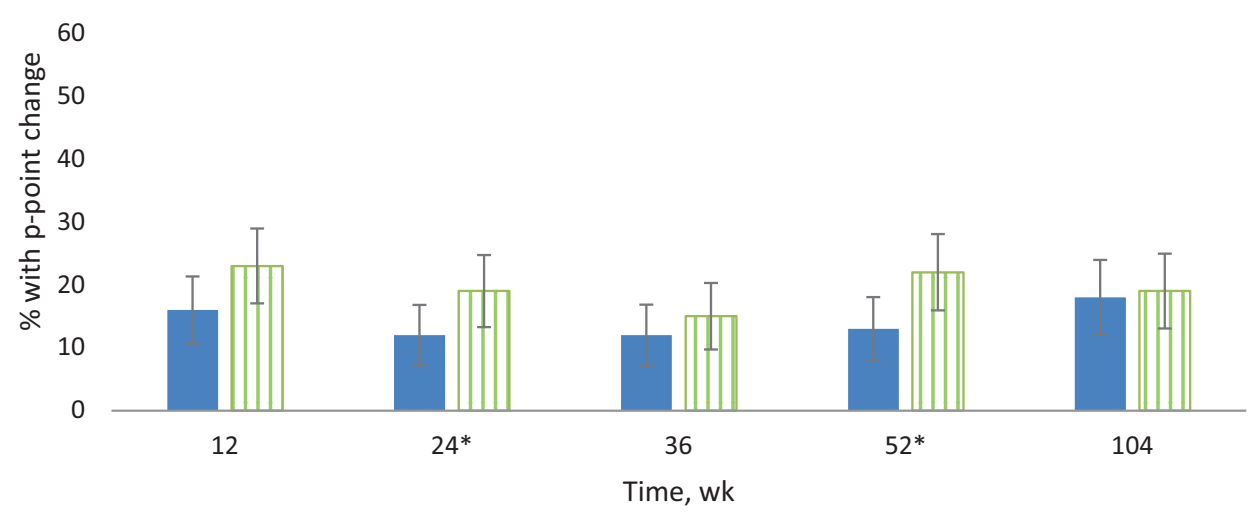

- Control $\square$ ALC

Figure 3. Percent of subjects in each arm with persistent neuropathy defined as five-point decrease from baseline (A) and 10-point decrease from baseline (B). Error bars represent $95 \%$ confidence intervals. ${ }^{*} \mathrm{P}<.05$ based on a two-sided chi-square test. ALC = acetyl-L-carnitine.

persistent CIPN. Previous studies have shown an association between diabetes and the development of CIPN (26); however, patients with known diabetes were excluded from this clinical trial, so this would not have confounded the results. In the current study, baseline weight was associated with persistent CIPN among patients randomly assigned to ALC but not placebo. The reason for this interaction is unknown. A prior study found both an association between obesity and the development of CIPN and that moderate to vigorous physical activity may be associated with decreased odds of developing CIPN (14).

The current study has several unique strengths, including the size of the sample, multicenter prospective data collection, a homogeneous breast cancer population with standard taxane exposure, and long-term follow-up. However, there are also several limitations. We did not collect any objective data to define CIPN such as quantitative neurosensory tests, tests of balance, or nerve conduction tests. However, as discussed above, prior studies have shown a strong correlation between the FACT-NTX and quantitative neurosensory tests (3). The study excluded patients with baseline diabetes and where the mean Performance Status was less than 1; therefore, the results may underestimate the rate of toxicity for patients with more comorbidities. In addition, we don't know if patients developed diabetes during the study, although given the study's randomized design, there is no a priori reason to believe the development of diabetes would differ by treatment arm. Finally, the patient-reported outcome did not differentiate between sensory, motor, or autonomic neuropathy.

In summary, we found that in addition to increasing CIPN at 24 weeks, patients treated with 24 weeks of ALC had increased CIPN over the two-year follow-up period compared with placebo. We also found that CIPN persists following chemotherapy and that the worsening symptoms remained clinically meaningfully in about $30 \%$ of breast cancer patients treated with adjuvant taxane-based therapy. This is of particular interest given the increased use of taxane-based therapy over time in breast cancer patients. Currently, there is a paucity of therapies for the treatment or prevention of CIPN despite multiple prospective randomized trials (1). The unexpected increase in CIPN from an agent presumed to 
Table 3. Association between baseline factors and five-point decrease in FACT-NTX score from baseline to year 1 and year 2

\begin{tabular}{|c|c|c|c|c|c|c|c|c|}
\hline \multirow[b]{2}{*}{ Baseline factor } & \multicolumn{4}{|c|}{ FACT-NTX 5-point decrease from baseline to year $1^{*}$} & \multicolumn{4}{|c|}{ FACT-NTX 5-point decrease from baseline to year $2^{*}$} \\
\hline & $\begin{array}{c}\text { No } \\
(\mathrm{n}=221)\end{array}$ & $\begin{array}{c}\text { Yes } \\
(\mathrm{n}=129)\end{array}$ & OR $(95 \% \mathrm{CI}) \dagger$ & $\mathrm{P} \dagger$ & $\begin{array}{c}\text { No } \\
(\mathrm{n}=206)\end{array}$ & $\begin{array}{c}\text { Yes } \\
(\mathrm{n}=121)\end{array}$ & OR $(95 \% \mathrm{CI}) \dagger$ & $\mathrm{P} \dagger$ \\
\hline \multicolumn{9}{|l|}{ Age, $y$} \\
\hline$<60$ & $170(66.7)$ & $85(33.3)$ & Ref & & $156(66.4)$ & $79(33.6)$ & Ref & \\
\hline$\geq 60$ & $51(53.7)$ & $44(46.3)$ & 1.74 (1.07 to 2.82$)$ & .02 & $50(54.3)$ & $42(45.7)$ & 1.67 (1.02 to 2.73$)$ & .04 \\
\hline \multicolumn{9}{|l|}{ Race } \\
\hline White & $182(62.5)$ & $109(37.5)$ & Ref & .76 & $177(65.1)$ & $95(34.9)$ & Ref & .23 \\
\hline Black & $20(64.5)$ & $11(35.5)$ & 0.94 (0.43 to 2.04$)$ & & $14(56.0)$ & $11(44.0)$ & 1.47 (0.64 to 3.37 ) & \\
\hline Other & 19 (67.9) & $9(32.1)$ & 0.73 (0.32 to 1.69$)$ & & $15(50.0)$ & $15(50.0)$ & 1.81 (0.84 to 3.87$)$ & \\
\hline \multicolumn{9}{|l|}{ Ethnicity } \\
\hline Non-Hispanic & $201(62.0)$ & $123(38.0)$ & Ref & & $190(62.9)$ & $112(37.1)$ & Ref & \\
\hline Hispanic & $20(76.9)$ & $6(23.1)$ & 0.49 (0.19 to 1.26$)$ & .14 & $16(64.0)$ & $9(36.0)$ & 0.95 (0.41 to 2.23 ) & .91 \\
\hline \multicolumn{9}{|l|}{ Weight in kg, mean (SD)‡ } \\
\hline Placebo & $77.4(19)$ & $78.9(18)$ & 1.02 (0.94 to 1.11$)$ & .64 & $77.0(19)$ & $78.6(18)$ & 1.02 (0.94 to 1.12$)$ & .61 \\
\hline ALC & $72.4(16)$ & $83.4(26)$ & 1.14 (1.05 to 1.24$)$ & .002 & $71.9(18)$ & $84.0(23)$ & 1.16 (1.06 to 1.26$)$ & $<.001$ \\
\hline \multicolumn{9}{|l|}{ Mastectomy } \\
\hline No & $96(60.8)$ & $62(39.2)$ & Ref & & $95(65.1)$ & $51(34.9)$ & Ref & \\
\hline Yes & $125(65.1)$ & $67(34.9)$ & 0.81 (0.53 to 1.26$)$ & .36 & $111(61.3)$ & $70(38.7)$ & 1.17 (0.75 to 1.85$)$ & .49 \\
\hline \multicolumn{9}{|l|}{ Regimen } \\
\hline $\begin{array}{l}\text { Q3 wk docetaxel for } 4 \\
\text { cycles }\end{array}$ & $52(62.7)$ & $31(37.3)$ & Ref & .91 & $53(67.1)$ & $26(32.9)$ & Ref & .75 \\
\hline Biweekly paclitaxel for & $42(66.7)$ & $21(33.3)$ & 0.83 (0.41 to 1.65$)$ & & $35(60.3)$ & $23(39.7)$ & 1.32 (0.65 to 2.68 ) & \\
\hline \multicolumn{9}{|l|}{$\begin{array}{l}6 \text { cycles or q3 wk doce- } \\
\text { taxel for } 6 \text { cycles }\end{array}$} \\
\hline Biweekly paclitaxel for & $57(62.6)$ & $34(37.4)$ & 1.02 (0.55 to 1.88$)$ & & $55(64.7)$ & $30(35.3)$ & 1.12 (0.59 to 2.14$)$ & \\
\hline \multicolumn{9}{|l|}{4 cycles } \\
\hline Weekly paclitaxel & 70 (61.9) & $43(38.1)$ & 1.04 (0.58 to 1.87$)$ & & $63(60.0)$ & $42(40.0)$ & 1.36 (0.74 to 2.51$)$ & \\
\hline $\begin{array}{l}\text { Baseline FACT-NTX Score, } \\
\text { mean (SD) }\end{array}$ & $41.0(4)$ & $40.5(4)$ & 0.98 (0.92 to 1.03$)$ & .34 & $41.1(4)$ & $40.3(4)$ & 0.95 (0.90 to 1.01$)$ & .09 \\
\hline \multicolumn{9}{|l|}{ Performance Status } \\
\hline 0 & $168(64.4)$ & $93(35.6)$ & Ref & & $156(64.5)$ & $86(35.5)$ & Ref & \\
\hline 1 or 2 & $53(59.6)$ & $36(40.4)$ & 1.26 (0.77 to 2.08$)$ & .35 & $50(58.8)$ & $35(41.2)$ & 1.29 (0.78 to 2.14$)$ & .33 \\
\hline \multicolumn{9}{|l|}{$\begin{array}{l}\text { Granulocyte colony- } \\
\text { stimulating factor }\end{array}$} \\
\hline No & $114(64.0)$ & $64(36.0)$ & Ref & & $102(61.4)$ & 64 (38.6) & Ref & \\
\hline Yes & $103(61.7)$ & $64(38.3)$ & 1.07 (0.69 to 1.66$)$ & .78 & $101(64.3)$ & $56(35.7)$ & $0.86(0.55$ to 1.36$)$ & .52 \\
\hline
\end{tabular}

*The outcome is only based on the time point in question and does not take into account variability between the time points. ALC = acetyl-L-carnitine; CI = confidence interval; FACT-NTX = Neurotoxicity component of the Functional Assessment of Cancer Therapy-Taxane; OR = odds ratio.

†Odds ratios and $P$ values were calculated using logistic regression and are adjusted for treatment group, for all factors except weight. Tests are two-sided.

‡OR is per 5 -kg increase in weight.

reduce CIPN does emphasize the need to better understand the mechanisms of toxicities in order to better identify effective means to prevent or treat them.

\section{Funding}

This study was supported by the Conquer Cancer Foundation/ Breast Cancer Research Foundation (DLH); Susan Komen Foundation (DLH); National Cancer Institute, Division of Cancer Prevention, grant CA037429 (SWOG).

\section{Notes}

The funders had no role in the design of the study; the collection, analysis, aor interpretation of the data; the writing of the manuscript; or the decision to submit the manuscript for publication.

\section{References}

1. Hershman DL, Lacchetti C, Dworkin RH, et al. Prevention and management of chemotherapy-induced peripheral neuropathy in survivors of adult cancers: American Society of Clinical Oncology clinical practice guideline. J Clin Oncol. 2014;32(18):1941-1967.

2. Hershman DL, Unger JM, Crew KD, et al. Randomized double-blind placebocontrolled trial of acetyl-L-carnitine for the prevention of taxane-induced neuropathy in women undergoing adjuvant breast cancer therapy. J Clin Oncol. 2013;31(20):2627-2633.

3. Hershman DL, Weimer LH, Wang A, et al. Association between patient reported outcomes and quantitative sensory tests for measuring long-term neurotoxicity in breast cancer survivors treated with adjuvant paclitaxel chemotherapy. Breast Cancer Res Treat. 2011;125(3):767-774.

4. Sparano JA, Wang M, Martino S, et al. Weekly paclitaxel in the adjuvant treatment of breast cancer. N Engl J Med. 2008;358(16):1663-1671.

5. Jones SE, Savin MA, Holmes FA, et al. Phase III trial comparing doxorubicin plus cyclophosphamide with docetaxel plus cyclophosphamide as adjuvant therapy for operable breast cancer. J Clin Oncol. 2006;24(34): 5381-5387.

6. Bao T, Basal C, Seluzicki C, Li SQ Seidman AD, Mao JJ. Long-term chemotherapy-induced peripheral neuropathy among breast cancer survivors: Prevalence, risk factors, and fall risk. Breast Cancer Res Treat. 2016;159(2): 327-333. 
7. Winters-Stone KM, Horak F, Jacobs PG, et al. Falls, functioning, and disability among women with persistent symptoms of chemotherapy-induced peripheral neuropathy. J Clin Oncol. 2017;35(23):2604-2612.

8. Zanville NR, Nudelman KN, Smith DJ, et al. Evaluating the impact of chemotherapy-induced peripheral neuropathy symptoms (CIPN-sx) on perceived ability to work in breast cancer survivors during the first year posttreatment. Support Care Cancer. 2016;24(11):4779-4789.

9. Cella DF, Tulsky DS, Gray G, et al. The Functional Assessment of Cancer Therapy scale: Development and validation of the general measure. J Clin Oncol. 1993;11(3):570-579.

10. Cella D, Peterman A, Hudgens S, Webster K, Socinski MA. Measuring the side effects of taxane therapy in oncology: The Functional Assesment of Cancer Therapy-Taxane (FACT-Taxane). Cancer. 2003;98(4):822-831.

11. Bates D, Mächler M, Bolker B, Walker S. Fitting linear mixed-effects models using lme4. J Stat Softw. 2015;67(1):1-48.

12. Seifried HE, Anderson DE, Sorkin BC, Costello RB. Free radicals: The pros and cons of antioxidants. Executive summary report. J Nutr. 2004;134(11): 3143S-3163S.

13. Norman HA, Butrum RR, Feldman E, et al. The role of dietary supplements during cancer therapy. J Nutr. 2003;133(11 suppl 1):3794S-3799S.

14. Greenlee H, Hershman DL, Shi Z, et al. BMI, lifestyle factors and taxaneinduced neuropathy in breast cancer patients: The Pathways Study. J Nat Cancer Inst. 2017;109(2):djw206.

15. Mols F, Beijers T, Lemmens V, van den Hurk CJ, Vreugdenhil G, van de PollFranse LV. Chemotherapy-induced neuropathy and its association with quality of life among 2- to 11-year colorectal cancer survivors: Results from the population-based PROFILES registry. J Clin Oncol. 2013;31(21):2699-2707.

16. Ezendam NP, Pijlman B, Bhugwandass C, et al. Chemotherapy-induced peripheral neuropathy and its impact on health-related quality of life among ovarian cancer survivors: Results from the population-based PROFILES registry. Gynecol Oncol. 2014;135(3):510-517.
17. Pereira S, Fontes F, Sonin T, et al. Chemotherapy-induced peripheral neuropathy after neoadjuvant or adjuvant treatment of breast cancer: A prospective cohort study. Support Care Cancer. 2016;24(4):1571-1581.

18. Fontes F, Pereira S, Castro-Lopes JM, Lunet N. A prospective study on the neurological complications of breast cancer and its treatment: Updated analysis three years after cancer diagnosis. Breast. 2016;29:31-38.

19. Rowinsky EK, Chaudhry V, Forastiere AA, et al. Phase I and pharmacologic study of paclitaxel and cisplatin with granulocyte colony-stimulating factor: Neuromuscular toxicity is dose-limiting. J Clin Oncol. 1993;11(10):2010-2020.

20. Rowinsky EK, Eisenhauer EA, Chaudhry V, Arbuck SG, Donehower RC. Clinical toxicities encountered with paclitaxel (Taxol). Semin Oncol. 1993;20(4 suppl 3):1-15.

21. Schneider BP, Li L, Radovich M, et al. Genome-wide association studies for taxane-induced peripheral neuropathy in ECOG-5103 and ECOG-1199. Clin Cancer Res. 2015;21(22):5082-5091.

22. Schneider BP, Zhao F, Wang M, et al. Neuropathy is not associated with clinical outcomes in patients receiving adjuvant taxane-containing therapy for operable breast cancer. J Clin Oncol. 2012;30(25):3051-3057.

23. Gogas H, Shapiro F, Aghajanian C, et al. The impact of diabetes mellitus on the toxicity of therapy for advanced ovarian cancer. Gynecol Oncol. 1996;61(1) 22-26.

24. Kus T, Aktas G, Kalender ME, et al. Taxane-induced peripheral sensorial neuropathy in cancer patients is associated with duration of diabetes mellitus: A single-center retrospective study. Support Care Cancer. 2016;24(3):1175-1179.

25. de la Morena Barrio P, Conesa MA, Gonzalez-Billalabeitia E, et al. Delayed recovery and increased severity of paclitaxel-induced peripheral neuropathy in patients with diabetes. J Natl Compr Canc Netw. 2015;13(4):417-423.

26. Hershman DL, Till C, Wright JD, et al. Comorbidities and risk of chemotherapy-induced peripheral neuropathy among participants 65 years or older in Southwest Oncology Group Clinical Trials. J Clin Oncol. 2016;34(25):3014-3022. 